
imaginaire

\title{
Bernhard Struck, Claire Gantet, Revolution, Krieg und Verflechtung 1789-1815
}

\section{Marita Gilli}

\section{(2) OpenEdition Journals}

Édition électronique

URL : https://journals.openedition.org/ahrf/11728

DOI : $10.4000 /$ ahrf. 11728

ISSN : 1952-403X

Éditeur :

Armand Colin, Société des études robespierristes

Édition imprimée

Date de publication : 1 septembre 2010

Pagination : 189-191

ISBN : 978-2-200-92633-5

ISSN : 0003-4436

Référence électronique

Marita Gilli, «Bernhard Struck, Claire Gantet, Revolution, Krieg und Verflechtung 1789-1815 », Annales historiques de la Révolution française [En ligne], 361 I juillet-septembre 2010, mis en ligne le 22 mars 2011, consulté le 24 avril 2022. URL : http://journals.openedition.org/ahrf/11728 ; DOI : https:// doi.org/10.4000/ahrf.11728

Ce document a été généré automatiquement le 24 avril 2022.

Tous droits réservés 


\title{
Bernhard Struck, Claire Gantet, Revolution, Krieg und Verflechtung 1789-1815
}

\author{
Marita Gilli
}

\section{RÉFÉRENCE}

Bernhard Struck, Claire Gantet, Revolution, Krieg und Verflechtung 1789-1815, WBG

Deutsch-Französische Geschichte, Band 5, Wissenschaftliche Buchgesellschaft, Darmstadt, 2008, 272 p., ISBN 978-3-53414-703-8, 69.9€

1 Cet ouvrage concerne deux événements qui sont d'abord dus à l'histoire française : la Révolution et la période napoléonienne et pose la question de savoir comment des événements nationaux peuvent être intégrés dans une perspective transnationale. Les deux problèmes principaux sont ceux de la périodisation et de l'espace. L'introduction commence par cette phrase devenue célèbre de Nipperdey: «Au début était Napoléon ». En effet, les auteurs pensent que ce n'est pas tellement le début de la Révolution française qui marque une césure en Allemagne, mais que ce sont plutôt les défaites contre les armées napoléoniennes, en particulier l'année 1806. La rupture de 1789 leur semble moins importante, même si beaucoup se sont intéressés à ce qui se passait en France. Il est incontestable que la Révolution a été un catalyseur dans la politisation de la presse qui avait commencé vers 1770. Mais elle n'a été une rupture dans aucun des domaines politique, social, culturel ou économique. D'autres dates de rupture sont beaucoup plus importantes comme la fin de la Guerre de Sept Ans en 1763, la mort de Frédéric II en 1786 et le début de la guerre en 1792. Une histoire francoallemande devrait plutôt commencer en 1801 ou 1803 ou encore 1806 avec la fin du Saint Empire. Néanmoins l'espace compris entre les deux dates de 1789 et 1815 marque la transition vers l'époque moderne et la phase culminante de ce que Kosellek a appelé la Sattelzeit (1770-1830/50). Le laps de temps choisi ici : 1789-1815 reste donc légitime. 
2 À côté du problème de la périodisation, il y a celui de l'espace. Déjà les délimitations de la France et de l'Allemagne sont problématiques. Ces notions existent chez les contemporains, mais on ne peut savoir ce qu'elles recouvraient exactement. Par ailleurs, l'Allemagne est divisée; on sait bien qu'il faut parler des Allemagnes, et les territoires allemands ont été influencés de façon très différente. Selon les auteurs, il est donc impossible à l'heure actuelle de faire un ouvrage d'ensemble sur l'histoire francoallemande, la seule possibilité étant de faire des études partielles. Plutôt qu'un manuel, leur ouvrage se veut un essai qui soulève des questions et ne s'estime pas définitif.

La première partie donne un coup d'œil encyclopédique et retrace à la fois l'histoire de la Révolution et ses répercussions en Allemagne. Le chapitre 1 concerne l'année 1800 et pose le problème de la perception des frontières : en effet, la région du Rhin qui est au centre des débats était alors pour les Allemands à la périphérie. Si, à l'heure actuelle, on sait bien où se situent la France et l'Allemagne, ce n'était pas le cas vers 1800. Dans la majorité des consciences, le Rhin devait être la frontière entre les deux pays, comme en témoigne l'aquarelle de Goethe qui figure en couverture de l'ouvrage. Par ailleurs, il y a peu de sentiment de la nation dans un Saint Empire divisé. Elle ne se définit guère que par la langue (encore que les dialectes dominent, M.G.) et la littérature. Le chapitre 2 concerne la France et l'Allemagne avant la Révolution et cherche à relativiser la dichotomie entre Révolution d'un côté et réformes de l'autre. Les chapitres 3 et 4 suivent la chronologie des événements depuis la décennie de la Révolution jusqu'à l'époque napoléonienne. La première phase de la Révolution a été généralement bien accueillie en Allemagne par les intellectuels. L'année 1789 est plutôt vue de façon positive, en particulier certains buts de la Révolution à savoir l'abolition du système féodal et la Déclaration des droits de l'homme. Beaucoup voient dans la Révolution le départ d'une nouvelle ère. Mais dès le début, on craint la souveraineté populaire et on redoute les violences. La Terreur entraînera le désenchantement de la plupart des Allemands, même quand ils s'étaient engagés dans la Révolution. Souvent des réformes prévues, comme le Allgemeines Landrecht sont retirées ou amoindries par crainte d'un transfert des idées révolutionnaires. En revanche, la suppression des couvents qui avait commencé dans les années 70 avec Joseph II s'accélère grâce à la Révolution. Dans les régions protestantes, on salue la nationalisation des biens de l'Église. Les transferts sont difficiles à apprécier en raison de la disparité des États allemands, mais de nombreuses sociétés se sont formées à l'image des clubs, même dans les régions non occupées. La partie sur la République de Mayence fait état du transfert de nombreux symboles révolutionnaires, comme le bonnet phrygien, l'arbre de la liberté etc... Contrairement à la thèse de $\mathrm{F}$. Dumont, les auteurs estiment à juste titre que ce ne sont pas seulement les intellectuels qui ont fait la Révolution de Mayence, mais qu'une grande partie de la population y a participé. Il ne s'agit pas pour eux seulement d'une révolution imposée de l'extérieur. Ils pensent, comme R. Reichardt que la situation révolutionnaire avoisinait celle de villes comme Tulle ou Marseille. Quand les troupes révolutionnaires reviendront en 1797, elles seront d'ailleurs bien accueillies. En tout cas, les régions rhénanes ont été au centre de l'osmose franco-allemande et le processus d'acculturation a laissé des traces pendant longtemps et ces pays ont résisté à la plupart des réformes de Stein. En ce qui concerne l'époque napoléonienne, les auteurs font état de deux interprétations: la première insiste sur l'effectivité et la capacité d'être adopté du régime napoléonien, surtout dans les États satellites. La seconde insiste sur les compromis que Napoléon a dû faire pour assurer sa domination. Les recherches récentes posent toujours la question : rupture ou continuité ? Certains 
se réfèrent à la célèbre phrase de Nipperdey, mais pour d'autres la rupture se fait dans les pays occupés où les Français travaillent avec des Allemands révolutionnaires, tout en étant bien conscients que les Rhénans ne sont pas vraiment devenus des Français et qu'on ne peut pas parler d'Allemagne française. L'année 1806 peut être considérée à la fois comme une fin et un commencement. Après l'accueil très positif de Napoléon à Berlin, l'occupation se fait sentir, mais selon les auteurs, la guerre aurait été une guerre des monarques plus que des peuples. Le caractère de "guerre de libération » aurait été seulement littéraire. Des auteurs comme Arndt ou Fichte auraient eu un public très limité et, dans ce public, des admirateurs de Napoléon. Même après 1806 on ne pourrait pas encore parler d'un véritable sentiment national. En revanche, Stein et Hardenberg vont tenter de réformer, préférant une révolution par en haut plutôt que par en bas. La dernière partie de ce chapitre est consacrée à l'après guerre. Que reste-t-il après 1815 ? Même si l'année 1815 représente une rupture dans les relations franco-allemandes, il reste des survivances de l'héritage révolutionnaire et napoléonien. En particulier, la question d'un gouvernement légitimé par une constitution reste centrale. Les mouvements de 1830 et 1848 sont la conséquence de cette période. La littérature se fait largement l'écho de ces événements (560 romans historiques, plus de 600 biographies) et il est incontestable que la figure centrale du souvenir est Napoléon. L'historiographie française de cette période, en particulier Thiers, est traduite et a une forte audience. En revanche, l'historiographie allemande, en dehors de Buchholz, met beaucoup de temps avant de reconnaître en Napoléon un rénovateur et un modernisateur de l'Allemagne.

La deuxième partie, plus méthodologique, s'intéresse au questionnement et aux perspectives. Constatant que cette période comprise entre 1789 et 1815 est la première aussi riche en interférences de nos deux histoires avec la guerre, l'occupation, mais aussi les contacts scientifiques et culturels, les auteurs affirment qu'elle a été très étudiée par l'historiographie. En France, ils notent de nombreux débats et controverses qui ont peu affecté l'historiographie allemande, les débats entre historiens français et allemands restant rares. L'historiographie allemande a basculé à partir des années 1970 d'une recherche surtout centrée sur les réformes prussiennes à une recherche sur les réformes des régions du Rhin. Le premier chapitre part du paradigme normatif de la modernisation qui aurait marqué les recherches sur la Révolution et Napoléon depuis les années 50, celle-ci ayant été en conflit avec les expériences, les identités et le souvenir. La domination napoléonienne a généralement été conçue comme une modernisation devenue nécessaire, mais ce concept de modernisation serait à relativiser fortement. En effet, on a beaucoup négligé l'histoire de la perception des événements par les populations au profit de l'interprétation des «guerres de libération » en tant que facteurs d'un nationalisme allemand dirigé contre la France. Or ce ne fut vrai que pour une minorité d'intellectuels et l'inimitié contre la France ne doit pas être exagérée, c'est plutôt la guerre que l'on déplore. Si on lit les récits des voyageurs, on voit que peu leur importait qui gouvernait, mais qu'ils s'intéressaient surtout aux résultats. On y chercherait en vain des sentiments nationalistes et l'envie que les territoires devenus français redeviennent allemands. Les recherches modernes ont repoussé loin dans le xixe siècle ces aspirations nationales. Le deuxième chapitre reprend le fil de l'expérience et interroge la chronologie de l'espace 1789-1815, son caractère marqué par l'époque et la conscience du temps en 1800. Le temps compris entre 1789 et 1815 représente-t-il une époque? Beaucoup voient dans la Révolution française un événement qui touche l'histoire du monde. Tant de choses s'étant produites en si peu de temps, on a eu généralement l'impression d'une accélération du 
temps. Le troisième chapitre étudie la guerre en tant que possible catalyseur ou déclencheur de l'idée de nation et du nationalisme. On peut constater que la guerre a changé de nature pendant cette période. Alors qu'elle était considérée comme un mal pendant les Lumières, on y voit de plus en plus un instrument nécessaire dans la lutte pour la liberté. Donc, à la fois, elle est justifiée et on rêve d'une union européenne de républiques qui rendrait les guerres inutiles (Kant, Fichte, Görres). Aussi bien en France qu'en Allemagne, le nationalisme aurait plutôt été une conséquence des guerres qu'une cause préexistante. Enfin, le dernier chapitre est une évaluation méthodique des outils d'une histoire transnationale : la comparaison, le transfert et l'histoire croisée qui sont différenciés par rapport à l'arrière-plan des éléments spécifiques de cette période et à développer. Le but étant de trouver quel outil conviendrait le mieux pour une histoire transnationale de cette époque. Les auteurs estiment le bilan des recherches fondées sur le transfert ambivalent: si elles ont ouvert de nombreux champs inexplorés, elles restent partielles et perdent donc de leur capacité d'explication analytique. Il est en effet rare qu'elles montrent comment des espaces proches et des sociétés mêlées peuvent s'influencer mutuellement de façon à changer. Ce concept devrait donc être développé et il faudrait distinguer entre transfert voulu et transfert imposé, surtout pendant la période napoléonienne. Une question que cette recherche ne s'est pas trop posée jusqu'ici est: dans quelles régions et sous quelles conditions la domination napoléonienne a pu s'imposer assez longtemps et sans grande résistance pour que le système français puisse être acculturé ? Les auteurs suggèrent que les approches dominantes de la comparaison et du transfert devraient être élargies par des prémisses à l'histoire transnationale telles que shared history ou histoire croisée. Des recherches ultérieures pourraient montrer si les notions d'histoire vécue ensemble ou d'histoire croisée peuvent apporter quelque chose.

On le voit, cet ouvrage pose des problèmes fondamentaux d'interprétation et de méthodologie. Il est complété par une abondante bibliographie respectueuse des différents courants, une table chronologique et un index des noms propres. 